CONTRIBUTION TO THE KNOWLEDGE ABOUT MINIMIZATION OF CYANIDE CONSUMPTION IN GOLD MINING; OXIDIZING SALTS, AERATION AND OVERMOLDING FOR CYANICIDE MINERALS

\section{CONTRIBUCIÓN AL CONOCIMIENTO SOBRE LA MINIMIZACIÓN DEL CONSUMO DE CIANURO EN LA MINERÍA DEL ORO; SALES OXIDANTES, AIREACIÓN Y SOBREMOLIENDA PARA MINERALES CIANICIDAS}

Received 09292019

Accepted 12152019

Published 12302019

Vol. 36, No.5, pp. 190-197, Nov./Dic.2019 Revista Boliviana de Química

36(5), 190-197, Nov./Dec. 2019

Bolivian Journal of Chemistry

DOI : $10.34098 / 2078-3949.36 .5 .1$

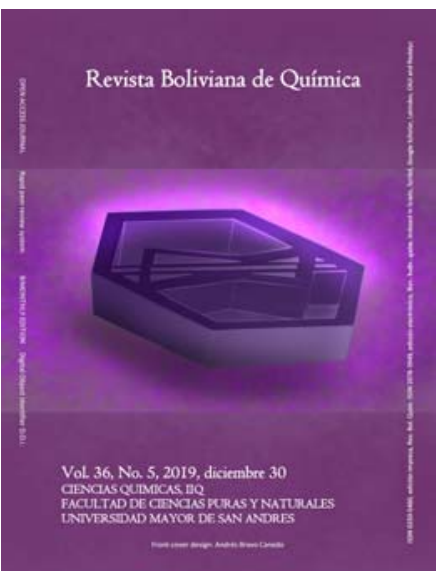

Roberto P. Huamaní B. ${ }^{1, *}$, Alberto F. Suero R. ${ }^{2}$, Luis Hau M. ${ }^{2}$, Pedro L. Mamani C. ${ }^{1}$, Paul K. Huanca Z. ${ }^{1}$, Victor B. Ascuña R. ${ }^{1}$, Stefany Cruz P. ${ }^{2}$, Yeimi L. Ochoa Q. ${ }^{2}$, Elizabeth V. Vega V. ${ }^{2}$

\begin{abstract}
${ }^{1}$ Escuela Profesional de Ingeniería de Procesos, Facultad de Ingeniería de Procesos FIP, Universidad Nacional de San Agustín de Arequipa UNSA, Av. Independencia s/n-Pab. Ing. Metalúrgica, pone +5154282813, Arequipa, Perú, metalurgia@unsa.edu.pe, http://fip.unsa.edu.pe/ingmetalurgica/

${ }^{2} \mathrm{H} \& M$ Chemical Group SAC, Prolongación Federico Recavarren N 1328-306, phone +51990260780, Lima 034, Perú, Ihaumendoza@hotmail.com
\end{abstract}

Keywords: Cyanidation, Gold, Oxidation, Passivation.

Palabras clave: Cianuración, Oro, Oxidación, Pasivación.

\title{
ABSTRACT
}

This research aims to contribute to the knowledge about the minimization of cyanide consumption in gold mining, mainly in complex minerals such as gold sulphides and gold auroarseniides; with the use of oxidizing salts. Cyanidation assays were performed varying the cyanide concentration, aeration, $\mathrm{pH}$ and particle size; salts of lead acetate and lead nitrate were used as passivants. In conventional cyanidation the reagent consumptions for these minerals are high; the use of passivating salts results in a reduction in the consumption of cyanide and other reagents, under the same control parameters and mineral overmolding process. Overmolding (closed milling circuit) is the process in which the mill uses a classifier whose thick product returns to the mill, and whose fine product passes to the next separation stage. This is applied after the metallurgical characterization of the sample by microscopy, observing the partial occlusion of the gold material in pyrrhotite matrix whose treatment requires high amounts of alkali and cyanide. The treatment with lead salts (passivation salts) that superficially alter the structure of 
the gold sulphide mineral was applied, with a decrease in the interaction with alkali and cyanide and consequent decrease in its consumption. As a result of the cyanidation process with salts, a gold solution of $97.78 \%$ was obtained for 24 hours, in significant contrast to the conventional treatment without passivation salts that gave a gold solution of $84.50 \%$. In another cyanidation experiment, a gold solution of $93 \%$ was obtained within 24 hours, from the ore pre-oxidized with air, while the auric solution was only $80 \%$ in the same period from ore without preoxidation.

In conclusion, a higher recovery yield of precious metal came from a pre-treatment of the mineral with passivation salts or by the aeration method, resulting in a good degree of particle release, with the consequent decrease in the consumption of cyanide and alkali.

\section{*Corresponding author: rhuamanibe@unsa.edu.pe}

\section{RESUMEN}

La presente investigación pretende aportar al conocimiento sobre la minimización del consumo de cianuro en la minería aurífera, principalmente en minerales complejos como ser sulfuros y arseniuros de oro; con la utilización de sales oxidantes. Se realizaron ensayos de cianuración variando la concentración de cianuro, la aireación, el pH y la granulometría; se utilizaron sales de acetato de plomo y nitrato de plomo como pasivantes. En la cianuración convencional los consumos de reactivos para estos minerales son altos; la utilización de sales pasivantes resulta en una reducción de consumo de cianuro y otros reactivos, bajo los mismos parámetros de control y proceso de sobremolienda mineral. La sobremolienda (circuito cerrado de molienda) es el proceso en el cual el molino utiliza en su trabajo un clasificador cuyo producto grueso regresa al molino, y cuyo producto fino pasa a la siguiente etapa de separación. Ésta es aplicada después de la caracterización metalúrgica de la muestra por microscopía, observándose la oclusión parcial del material aurífero en matriz de pirrotita cuyo tratamiento requiere de elevadas cantidades de álcali y cianuro. Se aplicó el tratamiento con sales de plomo (sales de pasivación) que alteran superficialmente la estructura del mineral sulfurado de oro, con una disminución de la interacción con el álcali y el cianuro y consiguiente disminución en su consumo. Como resultado del proceso de cianuración con sales se obtuvo una disolución de oro del $97.78 \%$ durante 24 hs, en significativo contraste con el tratamiento convencional sin sales de pasivación que dio una disolución de oro del $84.50 \%$. En otra experimentación de cianuración, se obtuvo una disolución de oro del $93 \%$ en 24 hs, a partir del mineral preoxidado con aire, mientras que la disolución áurica fue solo de un $80 \%$ en el mismo periodo a partir de mineral sin preoxidación.

En conclusión, un mayor rendimiento de recuperación de metal precioso provino de un pre-tratamiento del mineral con sales de pasivación ó por el método de aireación, dando como resultado un buen grado de liberación de la partícula, con la consiguiente disminución en el consumo de cianuro y álcali.

\section{INTRODUCCIÓN}

El metal precioso oro se halla asociado a minerales como la pirita, la pirrotita, la antimonita, y los arseniuros. El tratamiento metalúrgico para dichos minerales o concentrados se realiza en determinadas condiciones. Dichos procesos emplean cianuro entre otros, todos reactivos agresivos para el medio ambiente. La presente investigación pretende aportar al conocimiento sobre la minimización del consumo de cianuro en la minería aurífera, principalmente en minerales complejos como ser sulfuros y arseniuros de oro; con la utilización de sales oxidantes. El problema se aborda aplicando sales de plomo (sales de pasivación) que alteran superficialmente la estructura del mineral sulfurado de oro, con una disminución de la interacción con el álcali y el cianuro y consiguiente disminución en su consumo. Otra forma de atacar el problema es mediante una oxidación termoquímica que se realiza como un pre-tratamiento, podemos mencionar la pre-aireación con cal, la oxidación a presión, la tostación y la lixiviación bacteriana, entre otras [1]. Nagy [2] establece que el oro debe estar como partículas discretas y limpias ausentes de impurezas que puedan inhibir la reacción, y disponer de un adecuado suministro de oxígeno, para que se disuelva completamente en soluciones alcalinas de cianuro. En lo que concierne al segundo requerimiento, muchas minas de oro poseen constituyentes que se descomponen en soluciones de cianuro lo que ocasiona la aparición de compuestos, en soluciones que pueden inhibir la reacción de disolución del oro [1].

Demopoulus [3], Macassi [4], Ponciano [5] reportan que la refractariedad de las menas o concentrados es determinada por la resistencia que ofrecen estos materiales a la extracción directa de los metales preciosos por técnicas convencionales de cianuración alcalina. Esta refractariedad puede ser causada por: diseminación fina o Downloadable from: Revista Boliviana 191 de Química. Volumen 36 №5. Año 2019

http://www.bolivianchemistryjournal.org, http://www.scribd.com/bolivianjournalofchemistry 
Roberto P. Huamaní B. et al. RBQ Vol. 36, No.5, pp. 190-197, 2019

encapsulamiento de oro fino en pirita, pirrotita y/o arsenopirita, presencia de elementos carbonaceos (que precipitan al oro disuelto), presencia de cianicidas (consumidores de cianuro); y presencia de agentes consumidores de oxígeno

\section{Influencia del oxígeno}

El oxígeno o alguna sustancia oxidante es necesaria para ayudar a la disolución del cianuro; el oxígeno se une al hidrogeno que podría ser liberado y disminuye las pérdidas de cianuro en la forma de ácido cianhídrico gaseoso.

El más importante efecto de la extra aireación de la solución es disminuir el consumo de cianuro; teniendo en consideración que la solubilidad del oxígeno en la solución de cianuro alcanza alrededor de $8 \mathrm{mg} / \mathrm{L}, \mathrm{y}$ esta solubilidad no se altera por el aumento de la proporción de cianuro en la solución.

La proporción de la disolución del oro en el cianuro crece con la cantidad de oxígeno presente de aquí la necesidad de airear las soluciones.

La descomposición del $\mathrm{NaCN}$ es facilitada por la afinidad del $\mathrm{Na}^{+}$por el oxígeno libre, con lo cual queda en libertad el $\mathrm{CN}^{-}$naciente para unirse al oro [6-8].

\section{Efecto de las sales de $\mathrm{Pb}$}

Las sales de $\mathrm{Pb}$ (nitrato, acetatos) realizan dos funciones como agentes aceleradores: precipitar a los sulfuros solubles, y descomponer tioarsenitos o tioantimoniuros; en consecuencia el sulfuro de $\mathrm{Pb}$ al ser precipitado es posteriormente oxidado a tiocianato (sulfoarseniuro).

Los diversos compuestos de $\mathrm{Pb}$ formados como hidróxido, cianuro, plumbitos, etc, son ligeramente solubles en soluciones alcalinas de cianuro permitiendo adicionalmente precipitar más sulfuros y descomponer a tioarsenitos; entonces las soluciones de lixiviación quedan finalmente exentos de elementos nocivos como As y Sb [1].

\section{Efecto del As-Sb}

Muchas veces la presencia de As o Sb hace difícil o imposible la recuperación de los elementos auríferos, quedando ésta en niveles bajos, ésto debido a que estos elementos deterioran las soluciones perdiendo su poder lixiviante. El As y Sb se disuelven formando compuestos complejos de acción reductora que retardan o inhiben la disolución de $\mathrm{Au}$, entonces se necesita prevenir la disolución de estos o atenuar a los agentes de descomposición para controlar la arseniuracion.

Una manera de explicar los efectos retardadores del tioarsenito y de los tioantimonatos y sulfuros alcalinos en la disolución de $\mathrm{Au}$, es que los sulfuros formados durante la descomposición de los minerales de $\mathrm{As}$ y $\mathrm{Sb}$ en soluciones de $\mathrm{CN}^{-}$consumen el $\mathrm{O}_{2}$ necesario para facilitar la disolución del Au. Los arsenitos, antimonitos, tioarsenitos, tioantimonitos son reductores fuertes, debido a la formación de complejos. La descomposición de minerales de $\mathrm{As} \mathrm{y} \mathrm{Sb}$ resulta en compuestos o en sus iones, que se adhieren a la superficie expuesta del $\mathrm{Au}$ inhibiendo parcialmente su disolución.

Las alternativas para disminuir el efecto nocivo de los minerales cianicidas en varios procesos implican costos de tratamiento, presentándose las siguientes formas de solución: 1. La tostación parcial o total para formar $\mathrm{As}_{2} \mathrm{O}_{3}$ recuperando los gases de tostación para evitar la contaminación, 2. El control de la alcalinidad de solución ( $\mathrm{pH}=11)$ y 3. La adición de agentes pasivantes como son las sales de $\mathrm{Pb}$ con el $\mathrm{pH}$ adecuado [9].

\section{EXPERIMENTAL}

\section{Enfoque metodológico}

Se realizó una serie de pruebas estructuradas en etapas de acuerdo a la secuencia de tratamiento de muestras de la Fig. 1.

Las reacciones consideradas para tales fines fueron las siguientes:

- Cianuración con pre-tratamiento

- Cianuración con adicion de sales de plomo

- Cianuración con sobremolienda 


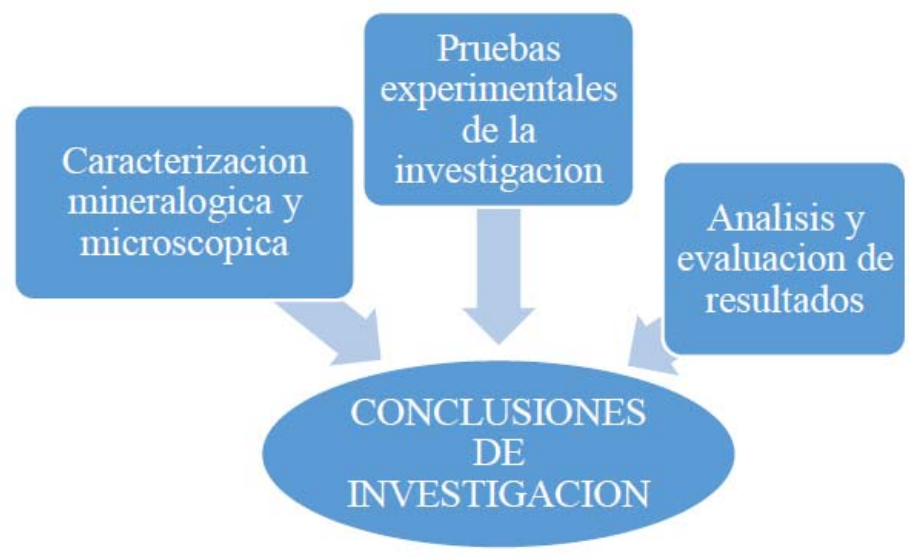

Figura 1. Secuencia del tratamiento de muestras. [Propia]

\section{Caracterización Microscópica}

El mineral utilizado para las pruebas fue caracterizado por un estudio microscópico determinándose la estructura del mismo, prediciéndose así su comportamiento durante el procesamiento.

En la Fig. 2 se puede observar por una parte, la asociación del elemento áurico con la pirrotita, y por otra, que la muestra árica se halla ocluida y semiocluida en los sulfoarseniuros. Esta última condición requerirá para su procesamiento, de un mayor grado de liberación de partícula (sobremolienda) para alcanzar un grado óptimo de recuperación.

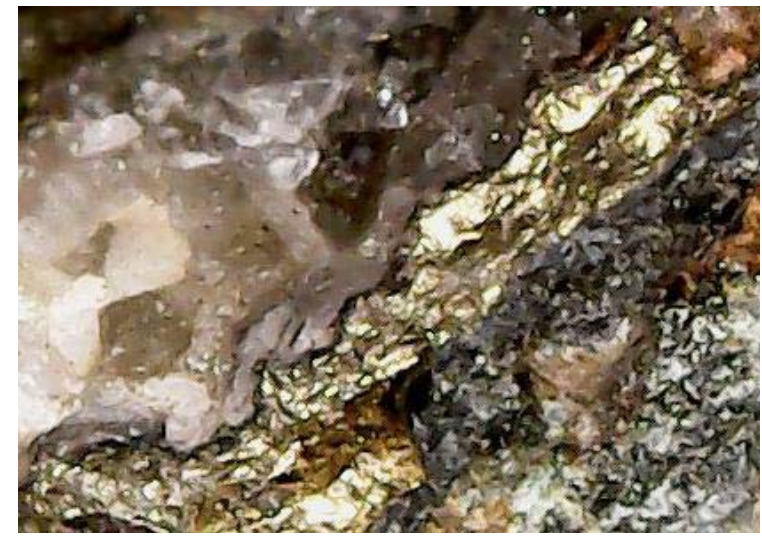

Figura 2. Vistas microscópicas del mineral de sulfuros. (Fuente: RHB/SCP-Mayo 2015)[8]

El carácter consumidor de oxígeno y cianuro de los sulfuros (cianicida) ha llevado a la proposición de las soluciones y la consecución de resultados que serán expuestos y discutidos en el presente artículo, esto, con el objetivo de proponer un control del consumo excesivo de cianuro.

El análisis microscópico condujo a la determinación del grado de liberación y la forma estructural del mineral, hecho que permitirá evaluar el efecto de los procesos metalúrgicos empleados.

\section{Lixiviación de minerales Auríferos}

La disolución del oro en medio cianurado en presencia de oxígeno ha sido la principal reacción para la recuperación del metal de sus menas [10]: 


$$
4 \mathrm{Au}+8 \mathrm{NaCN}+\mathrm{O}_{2}+2 \mathrm{H}_{2} \mathrm{O} \rightarrow 4 \mathrm{Na}\left[\mathrm{Au}(\mathrm{CN})_{2}\right]+4 \mathrm{NaOH}
$$

\section{Lixiviacion de minerales cianicidas}

La descomposición de la pirrotita por el $\mathrm{NaCN}$ es más enérgica que la de la pirita [11]:

$$
\begin{gathered}
\mathrm{Fe}_{7} \mathrm{~S}_{8}+\mathrm{NaCN}=\mathrm{NaSCN}+7 \mathrm{FeS} \| \mathrm{FeS}+4 \mathrm{O}_{2}=\mathrm{FeSO}_{4} \\
\mathrm{FeSO}_{4}+2 \mathrm{NaCN}=\mathrm{Fe}(\mathrm{CN})_{2}+\mathrm{Na}_{2} \mathrm{SO}_{4} \| \mathrm{Fe}(\mathrm{CN})_{2}+4 \mathrm{NaCN}=\mathrm{Na}_{4} \mathrm{Fe}(\mathrm{CN})_{6}
\end{gathered}
$$

El tratamiento de la pirrotita incluye el agregar nitrato de plomo y/o sales pasivantes de plomo en los molinos, según la reacción:

$$
\mathrm{Fe}_{7} \mathrm{~S}_{8}+2 \mathrm{PbO}=\mathrm{Pb}_{2} \mathrm{~S}+7 \mathrm{FeS}+\mathrm{O}_{2}
$$

Como ya se dijo, el hidróxido férrico tiene poca acción sobre el cianuro. El sulfuro ferroso se convierte en sulfato ferroso en la forma que ya conocemos, y si la alcalinidad es bien controlada, reacciona con el litargirio en la siguiente forma:

$$
\begin{gathered}
\mathrm{FeSO}_{4}+\mathrm{PbO}=\mathrm{PbSO}_{4}+\mathrm{FeO} \\
2 \mathrm{FeO}+3 \mathrm{H}_{2} \mathrm{O}+1 / 2 \mathrm{O}_{2}=2 \mathrm{Fe}(\mathrm{OH})_{3}
\end{gathered}
$$

Los diversos compuestos de $\mathrm{Pb}$ formados como hidróxido, cianuro, plumbitos, etc, son ligeramente solubles en soluciones alcalinas de cianuro permitiendo adicionalmente precipitar más sulfuros y descomponerse en tioarsenitos. Las soluciones de lixiviación quedan finalmente exentas de compuestos nocivos de As y Sb.

$$
\begin{gathered}
\mathrm{S}^{=}+\mathrm{Pb}^{++}=\mathrm{PbS} \\
2 \mathrm{AsS}_{3}^{-3}+3 \mathrm{~Pb}^{++}=\mathrm{PbS}+\mathrm{As}_{2} \mathrm{~S}_{3} \\
\mathrm{PbS}+\mathrm{CN}^{-}+1 / 2 \mathrm{O}_{2}+\mathrm{H}_{2} \mathrm{O}=\mathrm{CNS}^{-}+\mathrm{Pb}(\mathrm{CN})_{2}+2 \mathrm{OH}^{-} \\
\mathrm{PbS}+\mathrm{CN}^{-}+1 / 2 \mathrm{O}_{2}+2 \mathrm{OH}^{-}=\mathrm{CNS}^{-}+\mathrm{PbO}_{2}{ }^{=}+\mathrm{H}_{2} \mathrm{O}
\end{gathered}
$$

\section{Procedimiento}

Se dispuso de un mineral constituido por un complejo de sulfoarseniuros con mineral aurífero ocluido y semiocluido, con mínima presencia de oro al esto libre, asociado con una matriz de cuarcífera. Se realizó una serie de pruebas con distintos parámetros e insumos de acuerdo a los datos siguientes:

- Fuerza de cianuro $0.15 \%$

- Con $\mathrm{pH} 10.5$

- Dilución 3:1

- Tiempo 16 horas

- Grado de liberación de la partícula 100\% pasante malla 200 Tyler (74 micras); excepto la prueba con sobremolienda que fue del $100 \%$ pasante malla 250 Tyler (63 micras).

Para determinar el gasto de, y la presencia de cianuro libre después de las pruebas metalúrgicas, se realizó la titulación con nitrato de plata como titulante $(4.33 \mathrm{~g} / \mathrm{L})$ y como indicador ioduro de potasio al $5 \%$ en volumen.

\section{RESULTADOS Y DISCUSIÓN}

En las tablas 1 a 5 se observa los resultados de los balances metalúrgicos realizados. Una pre-aireación y posterior adición de sales de $\mathrm{Pb}$, permite el control y pasivación de los minerales cianicidas. La tabla 5 muestra comparativamente, la disminución del consumo de cianuro y del álcali utilizado. 


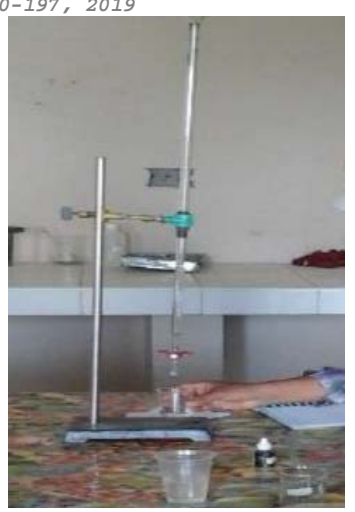

Figura 3. Titulación de solución cianurada.

Tabla 1. Balance metalúrgico de cianuración convencional

\begin{tabular}{lcccc}
\hline Productos & Pesos & Ensaye (g/Tm) & Cont. Met (g) & \% Recuperación \\
\hline Cabeza & $1.0 \mathrm{Kg}$ & 14.5 & 14.5 & 100.00 \\
Soluc. Pregnamt & $2.85 \mathrm{~L}$ & 4.0702 & 11.6 & 80.00 \\
Relave & $0.90 \mathrm{Kg}$ & 3.2222 & 2.9 & 20.00 \\
\hline
\end{tabular}

Tabla 2. Balance metalúrgico de cianuración con sales oxidantes

\begin{tabular}{lcccc}
\hline Productos & Pesos & Ensaye (g/Tm) & Cont. Met (g) & \% Recuperación \\
\hline Cabeza & $1.0 \mathrm{Kg}$ & 14.5 & 14.5 & 100.00 \\
Soluc. Pregnamt & $2.90 \mathrm{~L}$ & 4.6517 & 13.49 & 93.00 \\
Relave & $0.90 \mathrm{Kg}$ & 1.1222 & 1.01 & 7.00 \\
\hline
\end{tabular}

Tabla 3. Balance metalúrgico de cianuración con sobremolienda y sales oxidantes

\begin{tabular}{lcccc}
\hline Productos & Pesos & Ensaye (g/Tm) & Cont. Met (g) & \% Recuperación \\
\hline Cabeza & $1.0 \mathrm{Kg}$ & 14.5 & 14.5 & 100.00 \\
Soluc. Pregnamt & $2.90 \mathrm{~L}$ & 4.7241 & 13.70 & 94.50 \\
Relave & $0.88 \mathrm{Kg}$ & 0.9091 & 0.80 & 5.50 \\
\hline
\end{tabular}

Tabla 4. Balance metalúrgico de cianuración con sobremolienda, sales oxidantes y pretramiento

\begin{tabular}{lcccc}
\hline Productos & Pesos & Ensaye (g/Tm) & Cont. Met (g) & \% Recuperación \\
\hline Cabeza & $1.0 \mathrm{Kg}$ & 14.5 & 14.5 & 100.00 \\
Soluc. Pregnamt & $2.90 \mathrm{~L}$ & 4.8897 & 14.18 & 97.78 \\
Relave & $0.90 \mathrm{Kg}$ & 0.3556 & 0.32 & 2.22 \\
\hline
\end{tabular}

Tabla 5. Estudio comparativo de consumos de los tres sistemas de cianuración utilizados

\begin{tabular}{|c|c|c|c|c|}
\hline $\begin{array}{l}\text { Controles y } \\
\text { Parámetros }\end{array}$ & $\begin{array}{l}\text { Cianuración } \\
\text { Convencional }\end{array}$ & $\begin{array}{l}\text { Cianuración con } \\
\text { sales oxidantes }\end{array}$ & $\begin{array}{c}\text { Ciaanuración con } \\
\text { sobremolienda y sales } \\
\text { oxidantes } \\
\end{array}$ & $\begin{array}{c}\text { Cianuración con } \\
\text { sobremolienda, sales } \\
\text { oxidante y pretratamiento }\end{array}$ \\
\hline Cianuro & $6 \mathrm{Kg} / \mathrm{Tm}$ & $3.2 \mathrm{Kg} / \mathrm{Tm}$ & $2.8 \mathrm{Kg} / \mathrm{Tm}$ & $2.5 \mathrm{Kg} / \mathrm{Tm}$ \\
\hline OHNa (pH:11) & $3.1 \mathrm{Kg} / \mathrm{Tm}$ & $2.6 \mathrm{Kg} / \mathrm{Tm}$ & $2.2 \mathrm{Kg} / \mathrm{Tm}$ & $1.8 \mathrm{Kg} / \mathrm{Tm}$ \\
\hline Acetato de $\mathrm{Pb}$ & 0.00 & $0.11 \mathrm{Kg} / \mathrm{Tm}$ & $0.09 \mathrm{Kg} / \mathrm{Tm}$ & $0.09 \mathrm{Kg} / \mathrm{Tm}$ \\
\hline Aireación & Sin adición de aire & Sin adición de aire & Con adición de aire & Con adición de aire \\
\hline Molienda & Sin sobremolienda & Sin sobremolienda & Con sobremolienda & Con sobremolienda \\
\hline
\end{tabular}




\section{Análisis estadístico}

El análisis estadístico correspondiente a las pruebas de laboratorio se aprecia en las Figs. 4 y 5, que son gráficas de comparación:

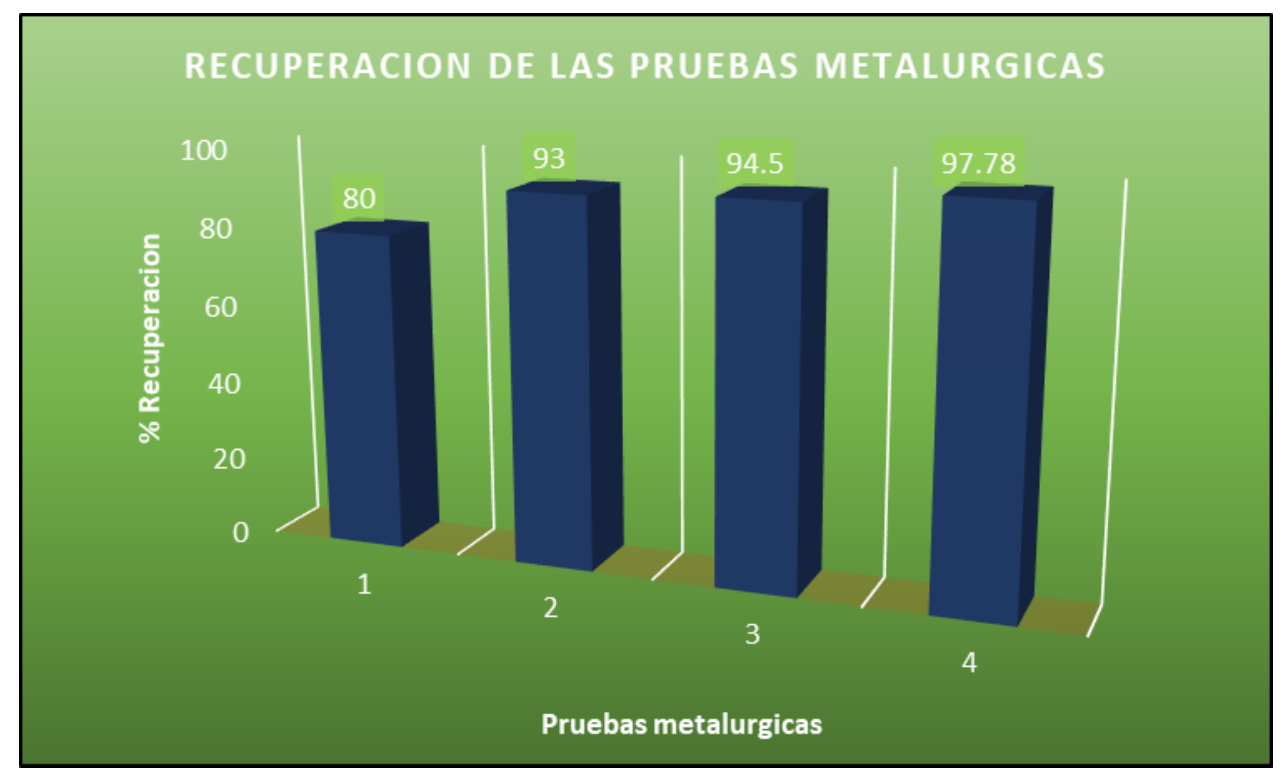

Figura 4. Recuperacion de pruebas metalurgicas.

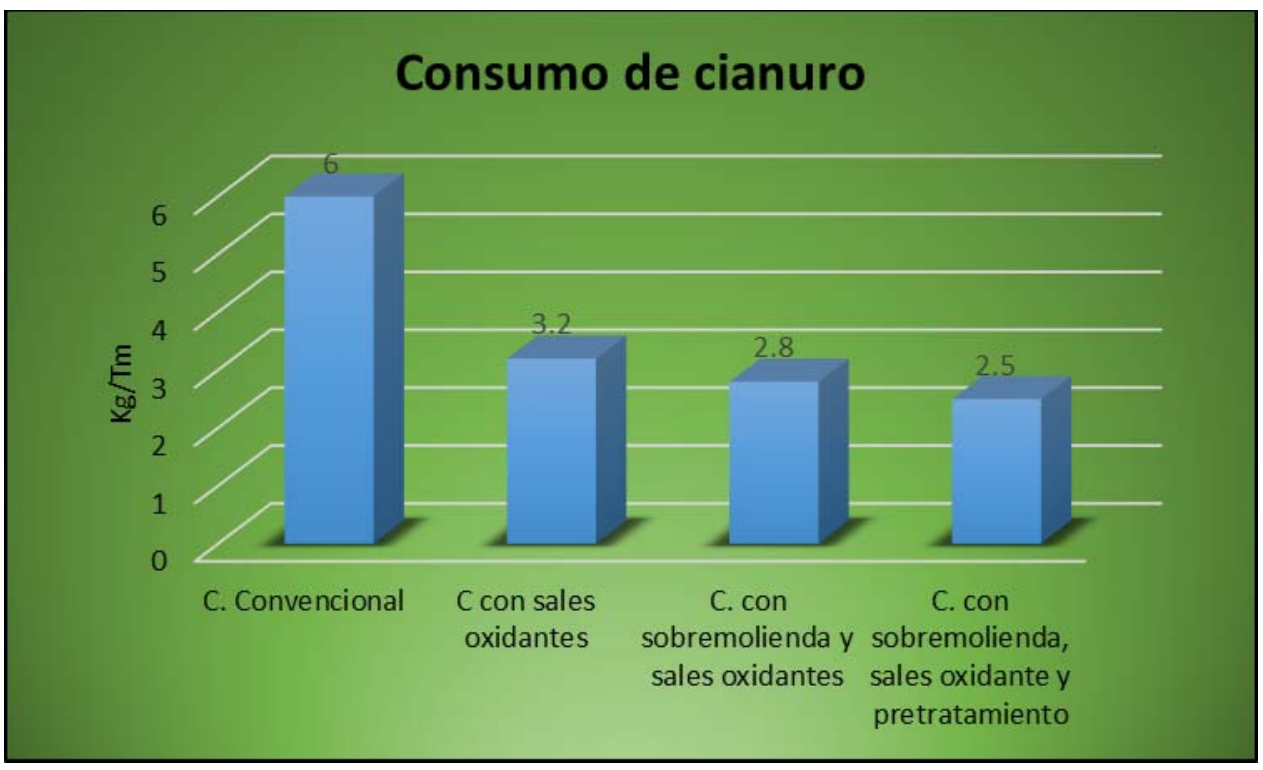

Figura 5. Consumo de cianuro de las pruebas metalurgicas.

La efectividad de la utilización de sales de plomo como agente inhibidor de la acción consumidora de álcali y de cianuro durante la cianuración quedó demostrada por el grado de acomplejamiento en medio hídrico de las sales de plomo con los sulfuros de hierro formando sulfuros estables con niveles máximos de oxidación.

Por otro lado, los sulfatos de hierro se estabilizan al acomplejarse y formar sulfatos de plomo con la generación de óxidos de hierro estables.

Downloadable from: Revista Boliviana 196 de Química. Volumen 36 №5. Año 2019

http://www.bolivianchemistryjournal.org, http://www.scribd.com/bolivianjournalofchemistry 
El aire adicionado en algunas pruebas permite que los sulfuros de hierro se oxiden y por ende no tengan el grado de ionización adecuado para poder consumir mayor álcali ni cianuro.

\section{CONCLUSIONES}

- Por medio de la caracterización mineralógica microscópica, se determinó la presencia estructural de pirita, pirrotita, arsenopirita, antimonita, especies de mayor importancia en la muestra de cabeza; además cuarzos y trazas de óxidos de hierro.

- Las sales de plomo permiten pasivar la superficie activa, principalmente de la pirrotita y de elementos ferruginosos activos que tienden a formar sulfuros ferrosos que son altamente activos (consumen oxígeno y cianuro) comportándose como elementos cianicidas que frente a la adición de sales de plomo quedan inhibidos.

- Los análisis comparativos demuestran la eficiencia del proceso utilizado, consiguiéndose una recuperación del $97.78 \%$ con pretratamiento y sobremolienda respectiva.

- Al realizarse un acondicionamiento de oxigenación conjuntamente con sales de plomo y una sobremolienda posterior antes de la cianuración convencional se permite reducir los costos de operación de la producción y una mejor eficiencia de recuperación del elemento aurífero.

\section{REFERENCIAS}

1. Aramburu Rojas, V.S. 2003, Modelo de la pre-aireación en concentrados de oro piritico, para la optimización del proceso de cianuración, Tesis Universidad Nacional Mayor de San Marcos. Lima-Perú. Universidad Nacional Mayor de San Marcos Facultad de Ingeniería Geológica, Minera, Metalúrgica y Geográfica, Unidad de Postgrado. Lima, Perú.

2. Nagy, I., Mrkusic, P., Mcculloch, H.W. 1966, Chemical Treatment of Refractory Gold Ores, Literature survey, Johannesburg, National Institute for Metallurgy, Report 38, 124.

3. Demopoulus, G. P., 1987, Mineralogical and Technological Aspects of Gold Extraction from Refractory Ores, Proc 20 Colloquia sur I'Or, CRM, 273-326.

4. Macassi, J. Y., Rivas, E. 1999, Nueva técnica hidrometalurgia, para mejorar la extracción de oro en materiales piriticos. I Simposio Internacional de Metalurgia. Tepsup Lima, Perú.

5. Ponciano, F. J., 1989, Tratamiento de materiales refractarios conteniendo metales preciosos por métodos convencionales y no convencionales. I Simposium Internacional de Metalurgia. Tepsup. Lima, Perú.

6. Miguel, E., Mihovilovic, D. 2001, Hidrometalurgia, fundamentos, procesos y aplicaciones, pp. 160-197.

7. Diez Canseco, E. 1974, Metalurgia del oro y la plata, Tesis Universidad Nacional de Ingeniería, Lima, Perú.

8. Sergio Missari, F.E. Metalurgia del Oro, Centro de Estudios y Promoción en Ciencias de la Tierra, $1^{a}$ Edición, 1993, Lima, Perú.

9. Huamani Bernal, R. 2002, Informe de investigación de cianuración de minerales sulfoarseniuros de Huancavelica, Arequipa, Perú.

10. Barsky, G, Swaison, S. J., Easley, N. 1935, Dissolution of gold and silver in cyanide solutions, Tras. Am. Inst. Min.-Metal. Engrs., 112660.

11. Arias Arce, V., Coronado Falcón, R., Santibañez Puente, L., Lovera Dávila, D. 2005, Refractariedad de concentrados auríferos, Revista del Instituto de Investigación FIGMMG, 8 (16), 5-14. 